\title{
Phage display screening identifies a novel peptide to suppress ovarian cancer cells in vitro and in vivo in mouse models
}

\author{
Cong Zhou ${ }^{1}$, Jiali Kang ${ }^{2^{*}}$, Xiaoxia Wang ${ }^{2}$, Wei Wei ${ }^{3}$ and Wenyan Jiang ${ }^{2}$
}

\begin{abstract}
Background: Ovarian cancer is a possibly lethal gynecological malignancy and this study utilized phage display technology to screen and identify peptides that specifically bind to ovarian cancer cells and explored the effects of these peptides on ovarian cancer cells in vitro and in vivo.

Methods: The phage displayed peptide library was used to isolate the peptides binding to and internalizing into the ovarian carcinoma cells. Positive phage clones were characterized with DNA sequencing and bioinformatics analysis and then validated with immunofluorescence. Subsequently, the selected peptides were investigated for their cancer-related functions, including cell adhesion, spreading, motility, and invasion in vitro and in vivo.

Results: Peptide1 read as SWQIGGNwas the positive peptide and showed preferential binding to the target cells. Peptide 1 also inhibited cell proliferation, migration, invasion and adhesion of ovarian cancer HO8910 cells in vitro. In vivo, Peptide 1 led to a lower tumorigenicity of HO8910 cells, which was characterized by the inhibitory effect on tumor growth and metastasis of ovarian cells.

Conclusion: These studies demonstrate that the phage display-identified tumor cell-binding peptide was able to control ovarian cancer cell viability, migration, invasion, and adhesion capacity in vitro as well as tumor growth and metastasis in vivo. Future studies will be aimed at evaluating the clinical efficacy of the peptide SWQIGGN in ovarian cancer patients.
\end{abstract}

Keywords: Phage display, Peptide, Ovarian cancer, Tumor cell viability, Invasion, Adhesion, Nude mouse tumor model

\section{Background}

Ovarian cancer is the most lethal gynecological malignancy and is characterized by insidious onset, rapid progression and poor survival [1, 2]. Indeed, most of ovarian cancer patients are diagnosed at an advanced stage of the disease with evidence of metastasis spreading beyond the ovaries [3] and the relapse rate of early stage ovarian cancer is up to $40 \%$ [4-7]. To date, the treatment of ovarian cancer is much like those of all other cancers and includes surgery, chemotherapy, radiation therapy and immunotherapy [8]. If the tumor is still confined in the ovary, surgery could be used to cure,

\footnotetext{
* Correspondence: kangjiali1961@21cn.com

${ }^{2}$ Department of Obstetrics and Gynecology, Guangzhou First People's Hospital, Guangzhou Medical University, Guangzhou 510180, China Full list of author information is available at the end of the article
}

while, for decades, paclitaxel and carboplatin chemotherapy have been used as the general standard of care for most ovarian cancer patients. However, tumor resistance does develop and certain subtypes of ovarian cancer are insensitive to chemotherapy. Radiation therapy is not effective for advanced stages of this disease and immunotherapy is just in the early stages of clinical trials [9]. Therefore, the identification of novel targets is urgently needed to develop innovative agents to effectively control ovarian cancer metastasis and progression [10]. To this end, recent studies reported that the secretion of cell surface proteins in human cancer cells could be especially important as therapeutic targets [11-13]. Our research is focused on identifying novel peptide-based probes using combinatorial chemistry and bacteriophage (phage) display to facilitate and identify novel cell 
surface molecules that could serve as biomarkers and therapeutic targets. As phage display is a useful technique we are able to examine protein-protein, protein-peptide, and protein-DNA interactions [14-16]. The "displayed" (selected) peptide is used to identify the receptor on the cell surface, which could be further used as a diagnostic marker or therapeutic target $[14,17]$. Previous studies utilized phage display peptide libraries to isolate and identify peptides that not only specifically bind to cell receptors in a cellspecific manner, but also exerted biological effects on the target cells [18-21]. Thus, we aimed to screen and identify peptides that could possess highly specific binding capabilities to human ovarian cancer cells, and then to investigate the potential of these selected peptides in terms of the control of ovarian cancer in vitro and in vivo. We carried out a series of experiments to explore whether such a peptide was effective in controlling ovarian cancer growth in vitro and in vivo. To the best of our knowledge, this study is the first report of such an approach to identify novel targets for ovarian cancer.

\section{Methods}

\section{Cell lines and culture}

A human ovarian cancer cell line, HO8910, was obtained from the Laboratory Animal Center, Sun-Yatsen University, Guangdong, China, and grown in Roswell Park Memorial Institute and cultured in 1640 medium (Invitrogen, Carlsbad, CA,, USA), supplemented with $10 \%$ fetal bovine serum (Hyclone, Logan, Utah, USA). The human ovarian epithelial cell line OSE was obtained from Xiandu Company (Guangzhou, China) and cultured in serum-free DMEM-F12 medium (Invitrogen) containing $10 \mathrm{mg} / \mathrm{ml}$ epidermal growth factor (Sigma, St Louis, MO, USA) according to a previously reported method [22]. These cells were incubated at $37{ }^{\circ} \mathrm{C}$ in a humidified atmosphere with $5 \% \mathrm{CO}_{2}$.

\section{Phage libraries and biopanning}

The Ph.D.-c7c phage display peptide library was purchased from New England Biolabs (Ipswich, MA, USA). This library contained approximately $1 \times 10^{13} \mathrm{pfu} / \mathrm{mL}$ phages with a diversity of $1.28 \times 10^{9}$ unique peptide sequences for up to 70 copies of each. Biopanning is an affinity selection technique for identifying peptides that bind to a given target [23]. In this study, the biopanning began with the incubation of the phage display peptide library with both normal and tumor cells, in which normal cells were used to deplete peptides that only bind to normal cells and then further incubated with tumor cells for identifying the peptides that only specifically bind to tumor cells. Briefly, $1 \mathrm{ml}$ of the primary phage display peptide library $\left(1 \times 10^{11} \mathrm{pfu} / \mathrm{ml}\right)$ in $5 \mathrm{ml}$ of phosphate buffered saline (PBS) was added to the confluent cell monolayer (normal ovarian epithelial cells, called depletor) in a $60 \mathrm{~mm}$ diameter dish and the cells were then incubated for $2 \mathrm{~h}$ on a rocker platform at $4{ }^{\circ} \mathrm{C}$. After that, the cell culture medium containing unbound phage was collected and transferred to another cell monolayer (HO8910 cells, called tester) and cells were incubated for another $2 \mathrm{~h}$ at $4{ }^{\circ} \mathrm{C}$. The cells were then intensively washed four times with Tris-based saline (TBS)-0.2 \%. Tween-20 and cell-bound phages were eluted from the cell surface by incubation with 1 mll of elution buffer [0.2 M Glycine-HCL pH 2.2] for $10 \mathrm{~min}$ at $4{ }^{\circ} \mathrm{C}$. The elution was immediately neutralized by the addition of $150 \mathrm{ml}$ of $1 \mathrm{M}$ Tris- $\mathrm{HCl}$ buffer $(\mathrm{pH} \mathrm{9.0)}$ and the aliquot of the eluted phage was used for microtitration, while the remaining phage was subjected to the next rounds of biopanning according to Wang JJ et al. [24]. for a total of four rounds of in vitro biopanning. At the end of each biopanning, the ratio of output $v s$. input phage numbers (number of cell-associated phage divided by the numbers of total phage applied to the cells) was calculated for each round.

\section{Enzyme-linked immunosorbent assay (ELISA)}

To identify phage clones, we performed an ELISA assay. Briefly, after four rounds of in vitro biopanning, twenty blue plaques were randomly chosen from the titration plate. HO8910 cells were plated into 96-well plates at a density of $10^{4}$ cells/well, washed and cultured in serum-free medium at $37{ }^{\circ} \mathrm{C}$ for $1 \mathrm{~h}$, and finally fixed in $4 \%$ paraformaldehyde for $20 \mathrm{~min}$ at room temperature. Cells were then washed three times with PBS-Tween 20 (PBS with $0.05 \% \mathrm{v} / \mathrm{v}$ Tween 20) and blocked with a blocking buffer (PBST containing $3 \% \mathrm{w} / \mathrm{v}$ BSA) for $1 \mathrm{~h}$. The selected phage clones $\left(10^{10} \mathrm{pfu} /\right.$ well $)$ and the negative control, M13KE phage (New England Biolabs) were added individually onto the cells and further incubated at $37^{\circ} \mathrm{C}$ for $1 \mathrm{~h}$. After that, the cells were washed three times with PBST and then cultured for $1 \mathrm{~h}$ in the presence of the HRP-conjugated anti-M13 antibody (Abcam, Cambridge, UK) at a dilution of 1:20 in the blocking buffer. The cells were then added to the Tetramethylbenzidine (TMB) working substrate solution ( $50 \mu \mathrm{L} /$ well; Sigma) and incubated for $20 \mathrm{~min}$ at room temperature. The incubation was stopped by adding $4 \mathrm{~mol} / \mathrm{L} \mathrm{H}_{2} \mathrm{SO}_{4}$. Finally, the absorbance was measured at $450 \mathrm{~nm}$ using a microplate reader (Bio-Rad model 550, Hercules, CA).

\section{Immunofluorescence staining}

Immunofluorescence staining was performed as described previously [25] to identify positive phage clones that bind to the cell surface. Briefly, after the 
cells were incubated with phage clones for $1 \mathrm{~h}$ at room temperature, the cells were washed with PBS and incubated with M13 antibodies at a dilution of 1:300 (Abcam) for $1 \mathrm{~h}$ at room temperature, Cells were subsequently washed with PBST and the second antibodies M13-FITC (Abcam) were added and incubated for $1 \mathrm{~h}$ at room temperature. The cells were finally observed using an inverted microscope, equipped with a digital camera and processed using the Viewfinder program. For each experiment, normal human ovarian epithelial cells were used as the negative control.

\section{DNA sequencing and peptide synthesis}

After four rounds of in vitro biopanning, twenty blue plaques were randomly chosen from the titration plate and amplified. Their ssDNA were extracted according to the instruction manual (Bioteke, Beijing, China). DNA sequencing analysis was performed by Shanghai Biotechnology (Shanghai, China) and the sequence data were analyzed by using the BLAST and PMOTIF programs. The candidate Peptide 1 (SWQIGGN, translated from the selected M13 phage DNA sequence) and an irrelevant control peptide (QFHFDAP) were synthesized and labeled with biotin by Shanghai Biotech Bioscience and Technology (Shanghai, China).

\section{Cell viability assay}

The in vitro effects of the selected peptides on cells were assessed using the MTT cell viability assay. Briefly, ovarian cancer HO8910 cells were plated into 96-well plates at a density of $10^{4}$ cells/well, in triplicate and grown overnight. The next day, $10 \mathrm{mM}$ of synthetic peptides were added into the cell culture wells at a final volume of $200 \mathrm{ml}$ of the regular growth medium/well. The irrelevant peptides were used as negative controls. At the end of each time point of the experiment, the media was removed and replaced with $20 \mathrm{ml}$ of $5 \mathrm{mg} / \mathrm{ml} \mathrm{MTT}$ (3\{4,5\}-dimethylthia-zol-2,5-diphenyl tetrazolium bromide (Sigma) in the growth medium, and the plates were further incubated in standard conditions for $4 \mathrm{~h}$. Afterwards, the supernatants of the cell culture were removed and replaced with $150 \mathrm{ml}$ dimethyl sulfoxide (DMSO) to solubilize the MTT dye. Absorbance was then determined using a Spectra max 96-plate reader at $490 \mathrm{~nm}$. Ovarian cancer HO8910 cells, without any treatment, were used as the blank group and DMSO was added to the control wells at equal volumes to those used for the test compounds.

\section{Cell invasion and migration assays}

The invasion and motility assays were performed using a Transwell chamber with a 8- $\mu \mathrm{m}$ pore size polycarbonate membrane (Corning, Hangzhou, China) as recommended by the supplier. For the invasion assay, the upper chamber of the polycarbonate filter was coated with $10 \mu \mathrm{l}$ of Matrigel (New England Biolabs) at a dilution of 1:3 with the growth medium. The chambers were then incubated at $37^{\circ} \mathrm{C}$ for $30 \mathrm{~min}$ to allow the Matrigel to form a continuous thin layer. The Peptide 1-HO8910, control peptide-HO8910, and HO8910 cells were starved for $24 \mathrm{~h}$ and then harvested. $5 \times 10^{4}$ cells in $200 \mu \mathrm{l}$ of $0.5 \%$ bovine serum albumin (Sigma) were placed in the upper chamber. The lower chamber was then filled with $600 \mu \mathrm{l}$ of growth medium subsidized with $20 \%$ fetal bovine serum. Cells were then cultured at $37{ }^{\circ} \mathrm{C}$ with $5 \% \mathrm{CO}_{2}$ for $48 \mathrm{~h}$. Afterwards, cells on the upper surface of the filter were removed using a cotton swab, whereas cells that invaded through the Matrigel filter to the lower surface were fixed with $4 \%$ neutral-buffered formalin and stained in $0.01 \%$ crystal violet solution. The cell numbers in five fields (up, down, median, left, and right. $\times 200$ ) were counted for each chamber, and the results were expressed as the mean $\pm \mathrm{SD}$. The tumor cell migration assay was performed in the same manner, with the exception that the filter was not coated with Matrigel.

\section{Cell adhesion assay}

To assess cell adhesion capacity after treatment with or without the peptides, we performed a cell adhesion assay. Briefly, the 96-well plate was first coated with $30 \mu \mathrm{l}$ of Matrigel at a dilution of 1:3 in PBS and incubated overnight with $0.1 \%$ BSA. Three groups of cells (the Peptide 1-HO8910, control peptide-HO8910, and HO8910 cells) were washed three times in serum-free medium, resuspended at a concentration of $1 \times 10^{6} \frac{\text { cells }}{\mathrm{ml}} / \mathrm{ml}$ in serumfree medium and added into each well at $100 \mu \mathrm{l} /$ well and the cells were then incubated at $37^{\circ} \mathrm{C}$ for $1 \mathrm{~h}$. Unattached cells were washed away with PBS. $10 \mu \mathrm{l}$ of the MTT solution was then added into each well and the plates were further incubated for 4 h. $100 \mu \mathrm{l}$ of DMSO was added to replace the growth medium to dissolve formazan by shaking for $10 \mathrm{~min}$. Absorbance values (A) were measured at a wavelength of $492 \mathrm{~nm}$ using a microplate reader. Results were expressed as the mean $\pm \mathrm{SD}$ and the adhesion rate was calculated using the formula: Relative adhesion rate $(\%)=\left(\mathrm{A}_{492}\right.$ of

Table 1 Specific enrichment of HO8910 cell-bound phages using an initial input of $10^{11} \mathrm{pfu}$

\begin{tabular}{llll}
\hline Round & Inputs (pfu/ml) & Outputs $(\mathrm{pfu} / \mathrm{ml})$ & Recovery rate $(\%)$ \\
\hline 1 & $2 \times 10^{11}$ & $0.9 \times 10^{5}$ & $4.5 \times 10^{-6}$ \\
2 & $2 \times 10^{11}$ & $3.4 \times 10^{6}$ & $1.7 \times 10^{-5}$ \\
3 & $2 \times 10^{11}$ & $4.4 \times 10^{7}$ & $2.2 \times 10^{-4}$ \\
4 & $2 \times 10^{11}$ & $2.2 \times 10^{8}$ & $1.1 \times 10^{-3}$ \\
\hline
\end{tabular}




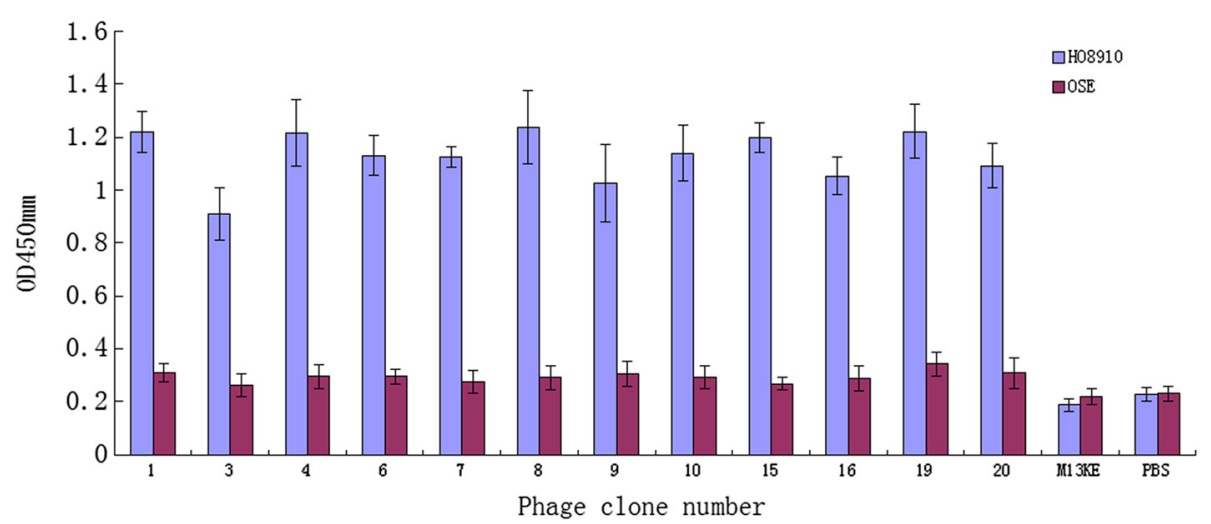

Fig. 1 Efficacy of phage clones in binding to HO8910 versus normal ovarian cells. HO8910 cells and OSE were incubated with phage display peptides. Unbound phages were removed by gentle washing and the bound phage clones were assayed by ELISA after incubation with HRP/ anti-M13 and then analyzed with a microplate reader at $450 \mathrm{~nm}$. The mean OD values represent activity of phage binding to cells. M13KE and PBS were used as controls. Lanes 1 to 12 depicts positive phage clones biopanned with HO8910 versus OSE cells

experimental group $/ \mathrm{A}_{492}$ of control group) $\times 100 \%$. Each experiment was repeated three times.

\section{Animal studies}

Twenty-one female athymic BALBC/c nude mice (4 to 6 weeks old with weights of $14.96 \pm 0.96 \mathrm{~g}$; animal approval number: 0113061) were obtained from Guangdong Medical Laboratory Animal Center, Guangzhou, China (SCXK(YUE)2008-0002) and maintained in Guangzhou Medical College Laboratory Animal Center, and were kept in specific pathogen free, temperature-controlled isolation conditions, and fed with sterilized food and autoclaved water. Animal breeding, care, and all experimental procedures were approved by the ethical and humane committee of Guangzhou Medical College and carried out strictly in accordance with the related regulations on administration of experimental animals under the SPF animal experiment number: 0052246. Specifically, these 21 mice were randomly divided into three groups: HO8910 cells preincubated with Peptide 1, HO8910 cells pre-incubated with the control peptide, and HO8910 cells without preincubations. Cells were then injected into the enterocoelia of each nude mouse with $1 \times 10^{6}$ cells in $200 \mathrm{ml}$ volume. The mice were then observed every two days and the abdominal circumference was measured. On day 42, the mice were euthanized and the tumors were dissected after the abdominal circumference and ascitic volume were measured. The tumor disseminated localization and the number of individual disseminated tumors were counted and the inhibitory rate was then calculated as follows: (control group average tumor weight - the average tumor weight)/control group average tumor weight] $\times 100 \%$. Tumor tissues were assessed for histopathology, immunohistochemistry and apoptosis.

\section{Immunohistochemistry}

To detect expression of vascular endothelial growth factor (VEGF) in tumor tissues from animal experiments, we performed immunohistochemistry using a rabbit polyclonal anti-VEGF antibody (Sigma, St Louis, MO, USA) according to the manufacturer's instructions. The anti-VEGF antibody was diluted to $1: 100$ and the biotinylated goat anti-rabbit IgG was diluted to 1:200. The staining results were evaluated by two independent investigators who were unaware of the identity of the tissue samples.

\section{Terminal deoxynucleotidyl transferase dUTP nick end labeling (TUNEL) assay}

TUNEL assay was used to detect apoptosis levels in tumor tissues using an in situ cell death detection kit conjugated with horseradish peroxidase (POD) (Roche Applied Science, Indianapolis, IN, USA) according to the manufacturer's instructions. The rate of apoptosis was evaluated by counting TUNEL-positive cells (brown-stained) and the apoptotic index was defined as the number of TUNEL-positive cells/total number of cells in 5 randomly selected high-power fields (magnification $\times 400$ ).

Table 2 Peptide identified for HO8910 cell binding (Amino Acid Sequences, deduced from DNA)

\begin{tabular}{lll}
\hline Clone & Peptide sequence & Incidence \\
\hline Phage 1, 3, 7, 16, 19, and 20 & SWQIGGN & 6 \\
Phage 4 & QFHFDAP & 1 \\
Phage 6 & TSPFWP & 1 \\
Phage 8 & TGNSNTQ & 1 \\
Phage 15 & TSHFEVP & 1 \\
Phage 9 and 10 & IGNSNTL & 2 \\
\hline
\end{tabular}



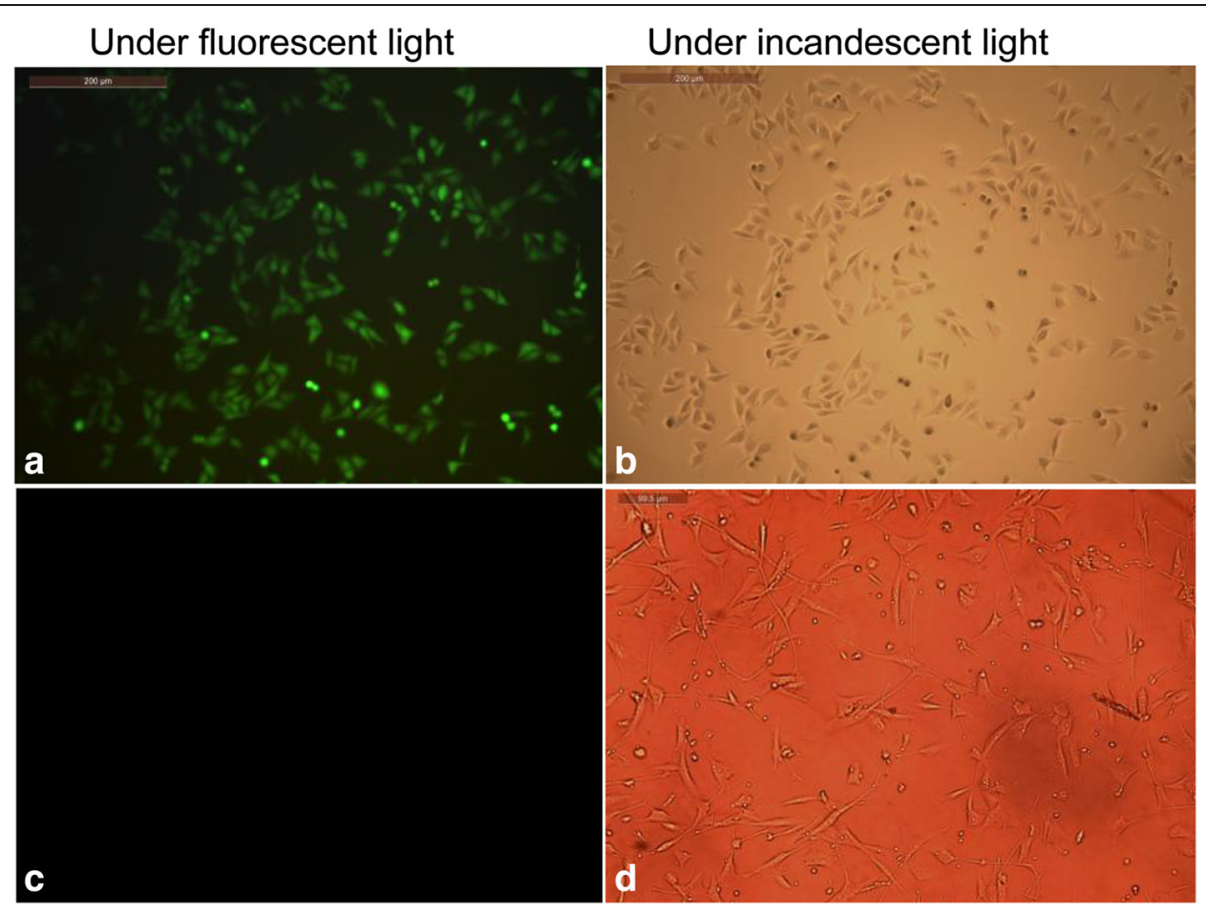

Fig. 2 Binding of Peptide 1 to the cell surface (×280). Immunofluorescence was used to stain Peptide 1 binding to HO8910 cells. Cells were visualized using a Leica DMRA2 fluorescence microscope. $\mathbf{a}$ and $\mathbf{b}$ HO8910 cells. $\mathbf{c}$ and $\mathbf{d}$ OSE cells

\section{Statistical analysis}

Statistical analysis was performed with SPSS 13.0 statistical software (SPSS Inc., Chicago, IL, USA). Results were expressed as the mean $\pm \mathrm{SE}$ of three or more observations (as indicated in each experiment). Differences amongst the three groups were assessed using one-way ANOVA and differences between the two groups were assessed using the Student-Newman-Keuls (SNK). A $p$ value equal to or less than 0.05 was considered statistically significant.

\section{Results and discussion}

Identification of phage peptides that specifically bind to the surface of ovarian cancer cells

In this study, we first screened and identified peptides that could specifically bind to the cell surface of ovarian cancer cells using the phage display assay. The input/ output phage ratio was calculated for each round of HO8910 cell biopanning and the ratio gradually rose and tended to stabilize after the fourth round. The data showed that the number of the fourth round phages

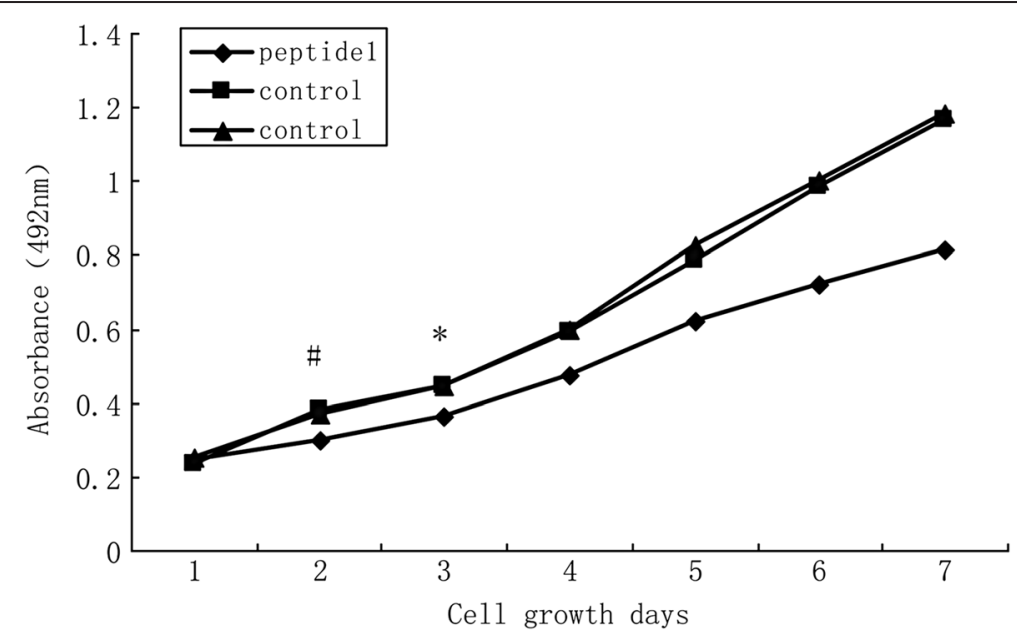

Fig. 3 Effects of Peptide 1 on the regulation of HO8910 cell viability. Ovarian cancer cells were treated with Peptide 1, negative control peptide, and blank control for up to 7 days and cell viability was assessed using a MTT assay. ${ }^{*} P<0.05$ 
were 244 times higher than that of the first round experiment (Table 1). After that, we performed ELISA to confirm the cell binding affinity of twenty selected phage clones. As shown in Fig. 1, 12 phage clones had very high affinity to bind to HO8910 cells compared to that of OSE cells. Thus, these 12 positive phage clones were propagated and ssDNA was extracted for DNA sequencing. After that, we identified unique DNA sequences that contained seven amino acids (Table 2). After similar peptide sequences had been aligned, most of the sequences revealed a consensus sequence at their amino terminal end and the peptide SWQIGGN appeared more frequently and then the peptides were blasted using the BLAST and PMOT IF programs to the Genbank database. However, there was no homology to any known protein that contained the phage peptide SWQIGGN, and thus, we named this clone Peptide 1.

We subsequently performed immunochemistry tests to further confirm the binding of Peptide 1 to the HO8910 cells. As shown in Fig. 2, Peptide 1 was able to

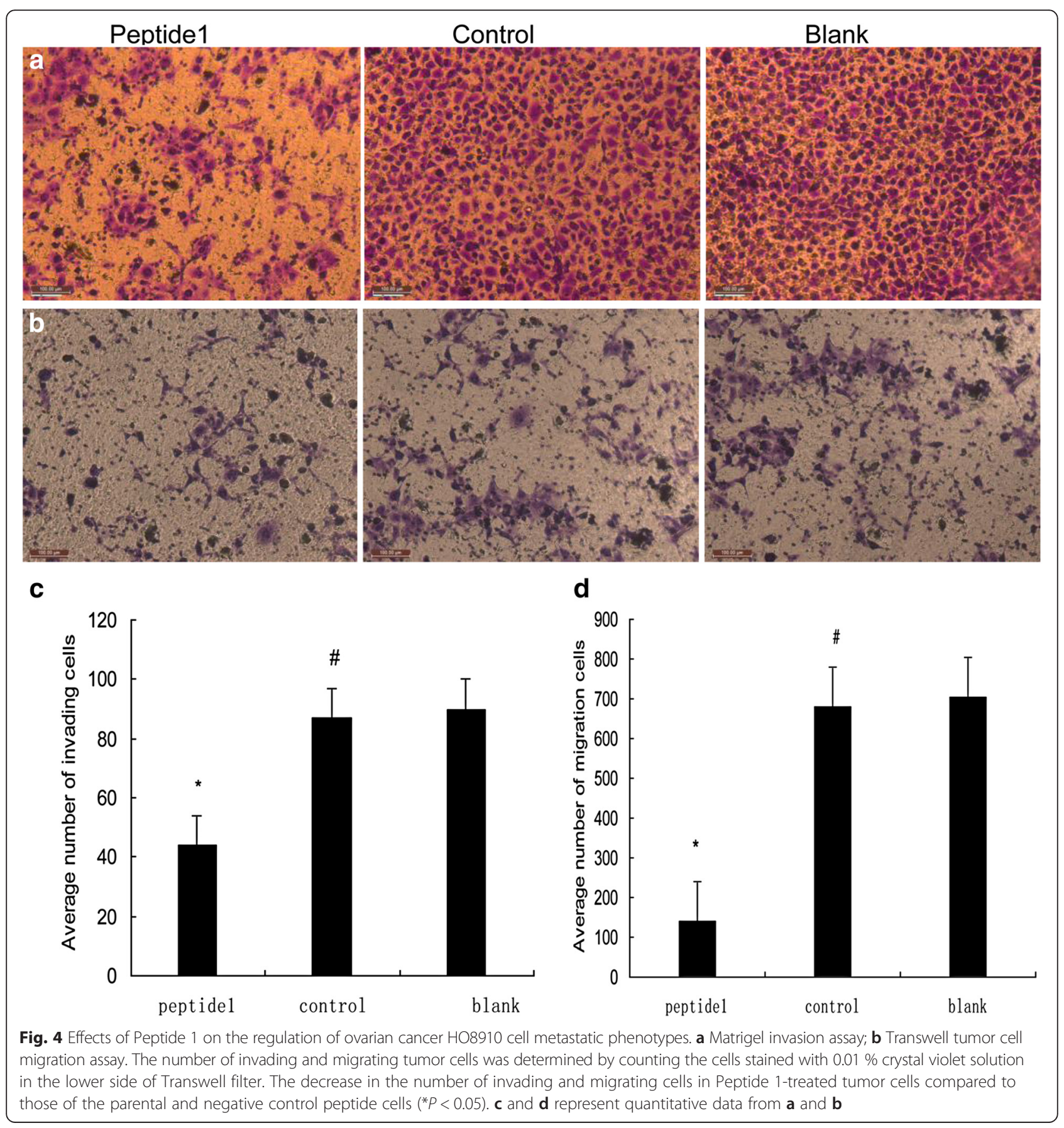


bind to HO8910 cells, but not normal human ovarian epithelial cells. Moreover, the negative control peptide clones did not significantly bind to both of the cell lines. These data indicate that Peptide 1 (i.e., SWQIGGN) was able to specifically bind to HO8910 cells.

\section{Effects of selected Peptide 1 on the regulation of ovarian cancer $\mathrm{HO} 8910$ cell biology in vitro}

We then assessed the effect of Peptide 1 on the regulation of ovarian cancer cell viability, apoptosis, invasion, and adhesion in vitro. As shown in Fig. 3, there were no differences in cell viability among the three treated groups at day 1 and $2(P>0.05)$; however, between day 3 and 6, Peptide 1 significantly reduced ovarian cancer cell viability $(p<0.05)$, but there were no differences in cell viability between the VCSM13-HO8910 and the blank controls $(p>0.05)$.

Furthermore, the tumor cell Transwell migration and invasion assay showed that HO8910-Peptide 1 had a pronounced reduction in both cell migration and invasion capacity compared to the negative peptide-treated and blank control cells $(p<0.05)$, whereas the negative peptide-treated and blank control cells showed similar migration and invasion capacity $(p>0.05$; Fig. 4$)$. These results indicated that Peptide 1 was able to block the metastatic potential of HO8910 cells in vitro.

In addition, we also assessed the effect of Peptide 1 on the regulation of HO8910 cell adhesion and found that Peptide 1 significantly blocked HO8910 cell adhesion capacity compared to the negative peptide-treated or bank control cells $(p<0.05)$, whereas the negative peptidetreated and blank control cells showed similar potential in terms of adhesion capacity ( $p>0.05$; Fig. 5$)$.
Effects of selected Peptide 1 on ovarian cancer H08910 cells in vivo

To further investigate whether Peptide 1 can control ovarian cancer in vivo, we treated ovarian cancer cells with Peptide 1 or negative control peptides for five days and then injected them into the enterocoelia of each nude mouse. As shown in Fig. 6, the abdominal circumference and mouse body weights were similar at the beginning of the experiment, but the abdominal circumference of HO8910-Peptide 1 cell-bearing mice didn't show much change two weeks after tumor cell injection, i.e., $65.8 \pm 2.1$ $\mathrm{mm}$, whereas the abdominal circumference of mice bearing HO8910-negative control peptide-treated and HO8910 parental cells was $86.0 \pm 3.4 \mathrm{~mm}$ and $85.4 \pm 4.2 \mathrm{~mm}$, respectively $(P<0.05)$. As shown in Table 3 , the volume of ascites, the number of tumors, the total number of disseminated tumor nodules and the tumor weight were all significantly smaller in the Peptide 1 treated cell group than those of the HO8910-negative control peptide and HO8910 parental cell groups $(p<0.05)$. However, no statistical significance in tumor growth was observed between control and HO8910 groups $(p>0.05)$. These results suggested that Peptide 1 was able to inhibit the growth and dissemination of ovarian cancer cells in this mouse transplantation model.

Effects of Peptide 1 on the regulation of VEGF expression and the apoptosis of ovarian cancer HO8910 cells in transplanted tumor tissues in mice

To further assess the effects of Peptide 1 on ovarian cancer cells in vivo, we analyzed the expression of VEGF protein in ovarian cancer HO8910 cell transplanted tumor tissues in mice. As shown in Fig. 7, immunohistochemical

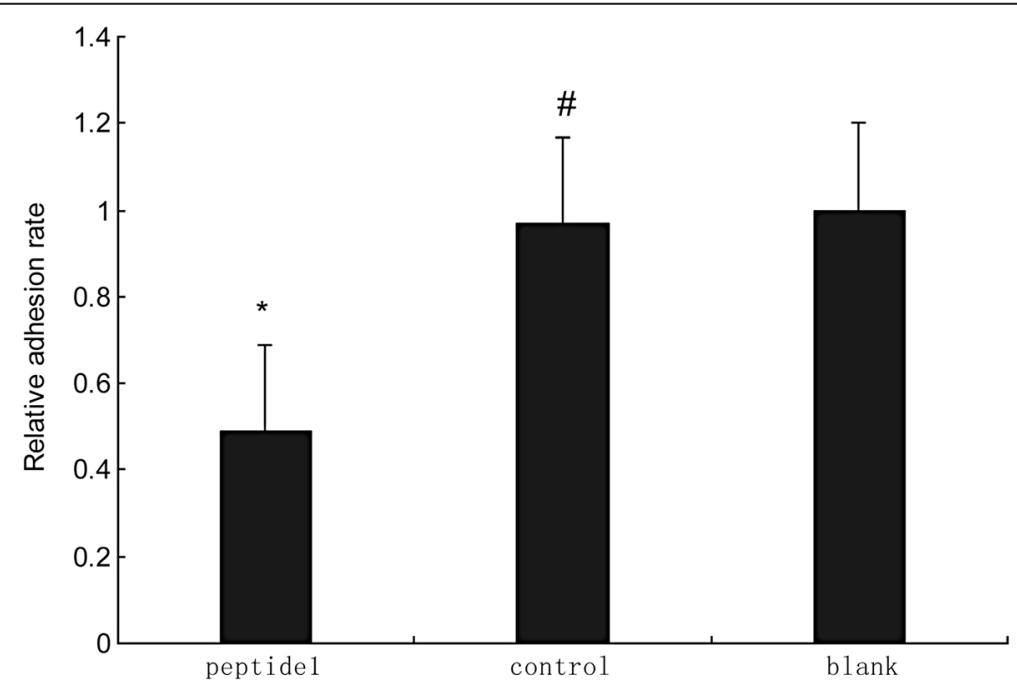

Fig. 5 Effect of Peptide 1 treatment on regulation of ovarian cancer cell adhesion capability. Ovarian cancer cells were treated with Peptide 1, negative control peptide, and blank control for five days and then the cells were seeded onto Matrigel-coated cell culture dishes and grown for $1 \mathrm{~h}$. Cells were washed with PBS and the remaining cells were visualized using MTT staining and quantified. ${ }^{*} P<0.05$ compared to the negative control peptide-treated cells and blank cells; \#P>0.05 versus blank cells 


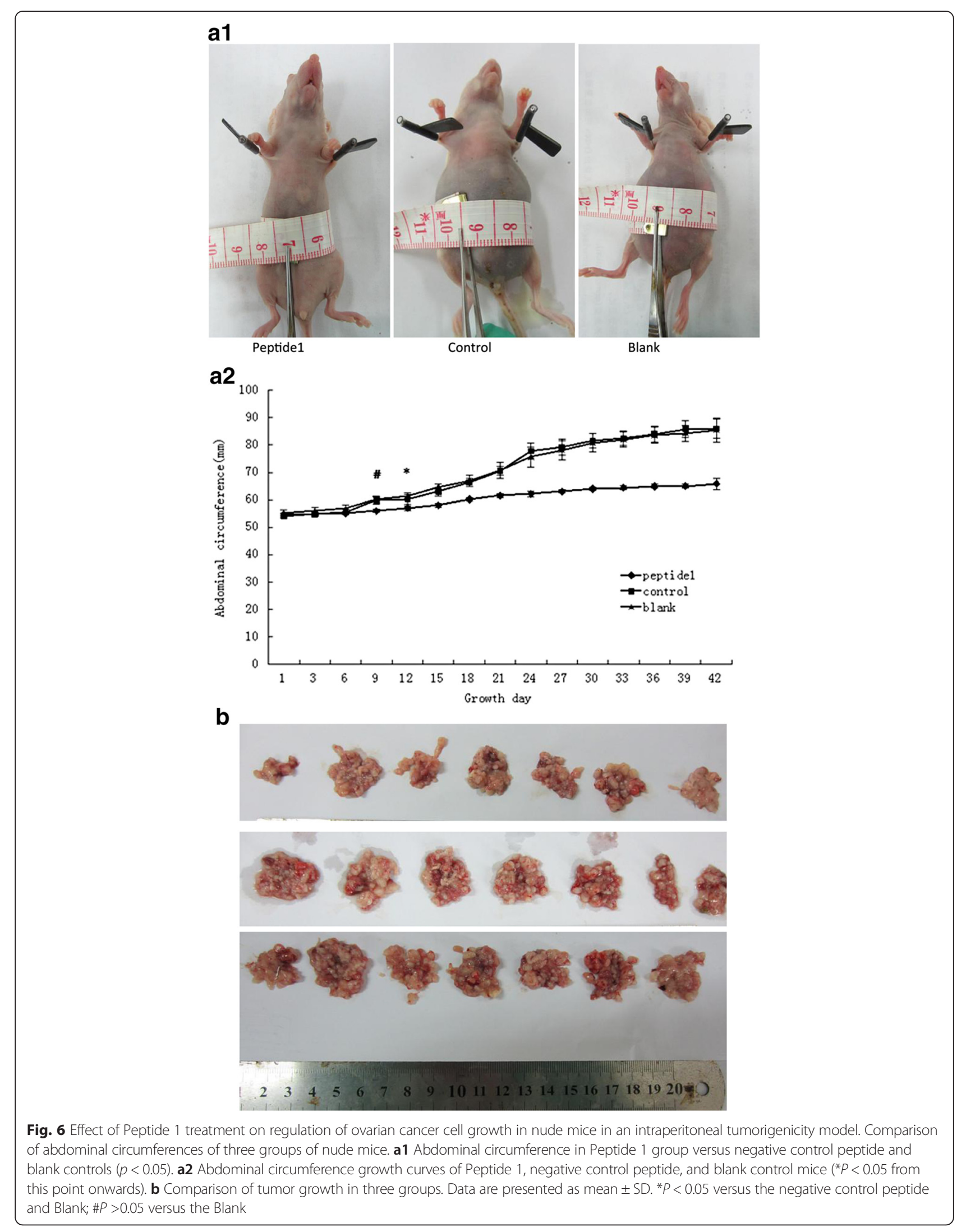


Table 3 Effect of peptide 1 on malignant behaviors of ovarian cancer xenografts in nude mice

\begin{tabular}{lllllll}
\hline Group & TFN & MTF & MR $(\%)$ & TV $(\mathrm{ml})$ & TW $(\mathrm{g})$ & IR $(\%)$ \\
\hline Blank & $7(7 / 7)$ & $103 \pm 26.40$ & 100 & $1.40 \pm 0.64$ & $1.77 \pm 0.44$ \\
Control & $7(7 / 7)$ & $94.86 \pm 30.62$ & 100 & $1.32 \pm 0.75$ & $1.67 \pm 0.37$ & - \\
Peptide 1 & $7(5 / 7)$ & $28.60 \pm 13.16^{*}$ & $71.4^{*}$ & $0.24 \pm 0.18^{\#}$ & $0.52 \pm 0.28^{*}$ \\
\hline
\end{tabular}

TFN tumor formation number, MTF metastatic tumor foci, MR metastatic rate, $T V$ tumor volume, $T W$ tumor weight, IR inhibitory rate; The values of metastatic tumor foci and tumor weight are presented as mean \pm SD of $n=7$ mice in relevant groups. ${ }^{*} P<0.001$ and ${ }^{*} p<0.05$ vs. the control and HO8910 groups

TFN, Five weeks post-inoculation, the nude mice were sacrificed, and then tumor formation was calculated for each mouse

$M T F$, collection of all tumors in each mouse, and then measurements of the number of tumors

MR, the MR of control = the MIF of control/ the MIF of blank; the MR of Peptide $1=$ the MIF of Peptide 1 /the MIF of blank;

$T W$, collect all the tumors from each mouse, and then their weights by scale

$I R$, IR of control $=[1$-(the weight of control/the weight of blank) $] * 100 \%$

IR of Peptide $1=\left[1\right.$-(the weight of Peptide 1/the weight of blank)] ${ }^{*} 100 \%$

staining data showed that the expression of VEGF protein in Peptide 1 treated ovarian cancer cells was markedly lower than in the control HO8910 cell group $(p<0.05)$, indicating that Peptide 1 could inhibit the expression of VEGF in HO8910 cells in vivo.

\section{Peptide 1 promoted cell apoptosis in vivo}

We then performed the TUNEL assay to detect the effect of Peptide 1 on the induction of cell apoptosis in tissue specimens from the nude mouse intraperitoneal tumorigenicity model. As shown in Fig. 8, the TUNELpositive nuclei were stained brown, and the apoptotic index was significantly higher in the Peptide 1 group compared to the control or HO8910 group $(p<0.05)$. However, there was no statistical significance observed between the control and HO8910 groups $(P>0.05)$. These results indicated that Peptide 1 promoted cell apoptosis in vivo.

Novel approaches could be used to identify and develop more effective therapeutic agents for the control of ovarian cancer in clinic. In this study, we utilized the phage display technique to screen and identify peptides that specifically bind to ovarian cancer cells to act as a therapeutic strategy. We identified 12 phage clones that could specifically bind to ovarian cancer cells but not normal ovarian epithelial cells. After DNA sequencing and blast search of Genbank, we found a novel peptide
SWQIGGN, which did not have an homology to any known protein. We then confirmed its ability to bind to ovarian cancer cells immunochemically and we explored the effects of this peptide on the control of ovarian cancer in vitro and in vivo. Our data showed that the treatment of ovarian cancer cells with this peptide significantly reduced tumor cell viability and suppressed tumor cell migration, invasion, and adhesion capacity. In vivo nude mouse studies showed that peptide treated-ovarian cancer cells had much smaller ascite volume, a smaller number of disseminated tumor nodules, and smaller tumor total weights than those of negative control peptide-treated HO8910 $(P=0.0035)$ and parental HO8910 $(P=0.0087)$ cells. Treatment with this peptide also inhibited VEGF expression. Thus, our current data identified a phage display-identified tumor cell-binding peptide that was able to control ovarian cancer cell viability, migration, invasion, adhesion capacity in vitro and tumor growth and metastasis in vivo. Future studies are needed to evaluate its efficacy and side effect profiles before it can be translated into clinical applications for the treatment of ovarian cancer patients.

The phage display technique is a useful tool to identify short peptides or antibodies that can specifically bind to and regulate functions of target proteins and the latter could be used for early tumor diagnosis, vaccines and as therapeutic targets. If proteins are expressed on the cell

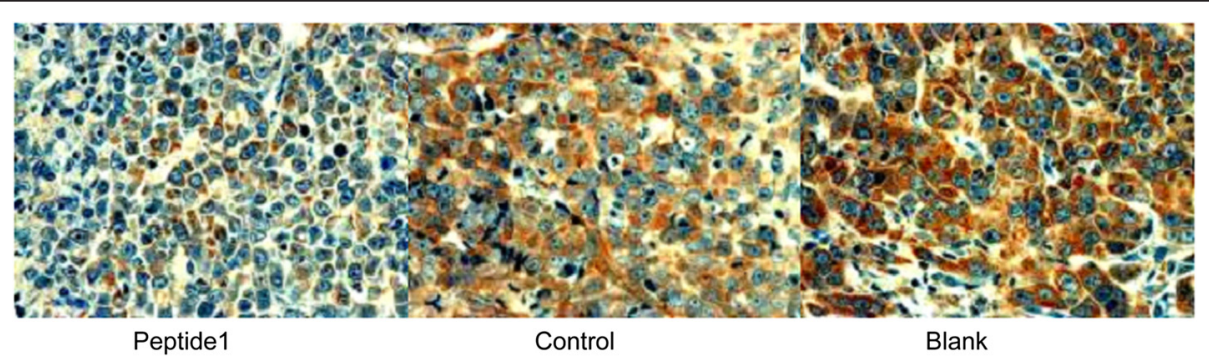

Fig. 7 Effect of Peptide 1 treatment on regulation of VEGF expression in nude mice in an intraperitoneal tumorigenicity model ( $\times 400)$. Mouse tumor tissues were dissected and immunostained with anti-VEGF antibody. Stained tissue sections were reviewed and scored (see Methods section). Expression of VEGF in mouse tumor tissues of Peptide 1-treated tumor cells was markedly decreased compared to mouse tumor tissues of the negative control peptide-treated tumor cells and parental HO8910 cells 


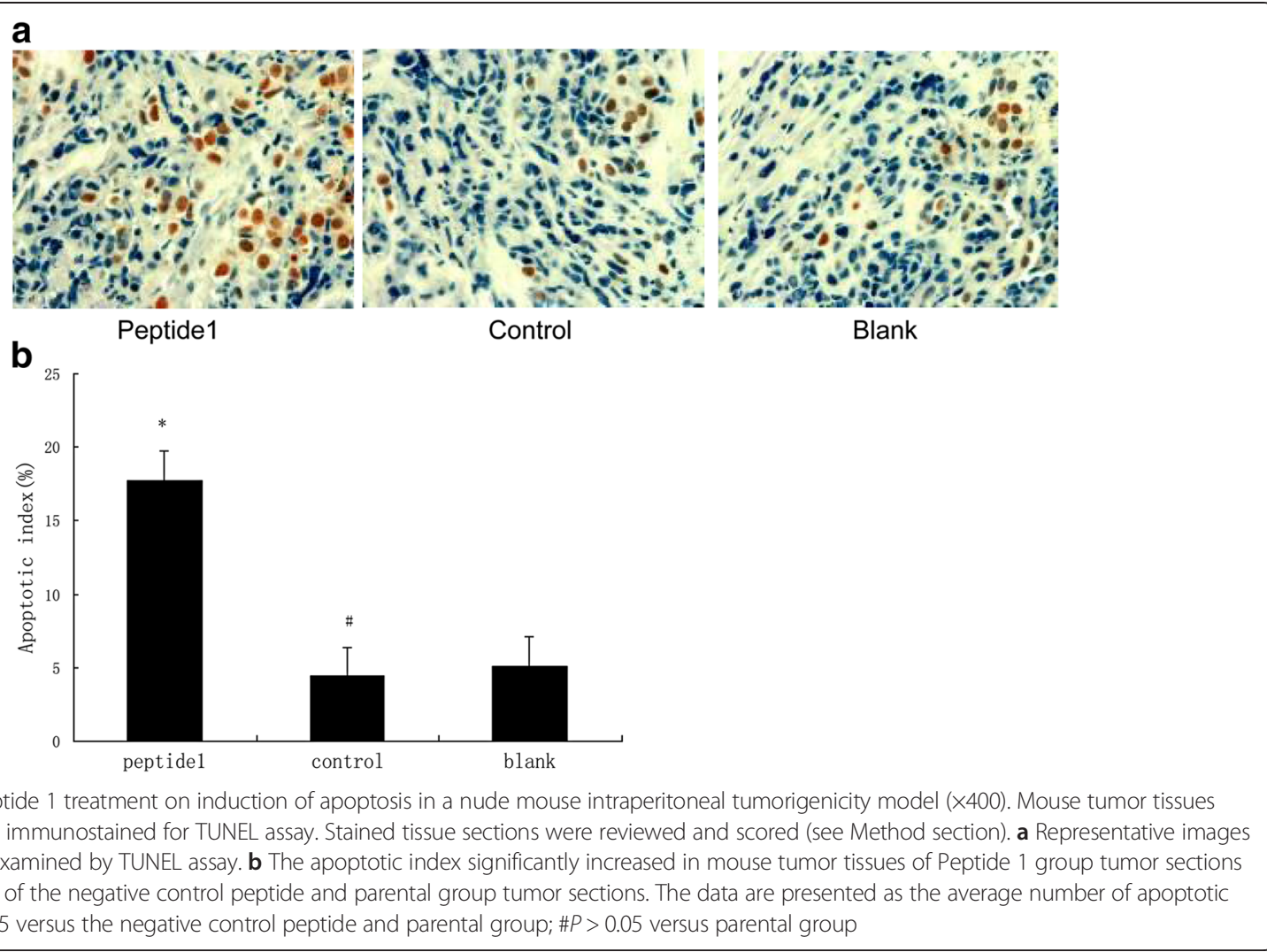

surface, they could also be useful for the delivery of bioactive agents, such as small-molecule drugs and radionucleotides to specifically target the cells which express these proteins [26]. Recently, this technology was utilized to screen prostate and breast cancer cell-specific functional peptide ligands [27]. Our study identified a group of ovarian cancer cell-specific binding peptides and we chose one of these peptides for further study as a therapeutic agent for ovarian cancer in vitro and in vivo. The data demonstrated the usefulness of this technique to screen and identify tumor cell specific ligands.

In this study, we first biopanned our phage libraries with normal ovarian epithelial cells in order to remove the peptides that can bind to normal cells and then biopanned the remaining phage libraries with ovarian cancer cells to identify peptides that specifically bind to ovarian cancer cells. We obtained the ratio of output to input with an enrichment of 244 times in the last round compared to the first round. This study differed from that of other studies [28] in that they reported their data by selected cell surface-bound peptides to specifically bind to ovarian cancer cells but not stratified by normal cells. After that, we selected 20 phage clones for confirmation and 12 of them were shown to specifically bind to tumor cells versus normal cells. DNA sequencing and a blast search of Genbank identified a novel peptide SWQIGGN that didn't show any homology to any known protein. Thus, this peptide could be useful in the control of ovarian cancer; therefore, we then assessed the effects of this peptide on affecting the biological behaviors of ovarian cancer cells. The data showed that this peptide was able to reduce tumor cell viability, migration, invasion, and adhesion capacity. However, to date, it is unclear how this peptide blocked biological cell behavior. Future studies are needed to identify the protein or cell structure that this peptide binds to. However, since this peptide binds to a surface protein on tumor cells, such a protein could be a cell growth factor or hormone receptor and the binding of this peptide blocks the ligands-binding to the protein and therefore, inhibits their signaling transduction leading to the suppression of tumor cell growth, migration and adhesion capacity. Negative controls (non-specific) peptides used in the current study didn't show much activity in ovarian cancer cells. This negative control (i.e., IGNSNTL) was obtained after the fourth screening (Table 2).

Moreover, cell motility and tumor cell invasion are major components in the multistage process of cancer metastasis $[29,30]$. It is demonstrated that the in vivo blockade of tumor cell spreading can suppress tumor cell movement and invasiveness in vitro. Our current data showed that this peptide inhibited ovarian cancer cell adhesion, migration, and invasion capacity in vitro. 
Our nude mouse experiment results complimented our in vitro data, showing that peptide-treated ovarian cancer cells had smaller tumor numbers and size, and metastasis lesions in nude mice. At the gene level, this peptide inhibited VEGF expression in tumor tissues. Indeed, tumor angiogenesis is the key to facilitate tumor growth and metastasis [2, 31]. However, it is unknown whether this peptide did block tumor angiogenesis by downregulation of VEGF expression. VEGF is the most important vascular growth factor [31] and its expression is regulated by different factors [32]. Thus, future studies will be needed to explore how this peptide reduces VEGF expression.

\section{Conclusions}

Our current study identified a novel peptide (SWQIGGN) that can 1) specifically bind to ovarian cancer cells and 2) control ovarian cancer in vitro and in vivo. However, our current study is just proof-of-principle and more thorough studies need to be performed before this peptide can be used in a clinical setting. Our future studies will focus on: 1) understanding the underlying mechanism of its action in ovarian cancer cells and identifying binding protein(s), 2) exploring the specificity of this peptide in other ovarian cancer cell lines versus other types of cancer cells and 3) assessing the clinical efficacy and side effects of this peptide in ovarian cancer patients.

\section{Abbreviations}

Al: apoptotic index; Anti-M13: Antil-M13 monoclonal antibody; BSA: bovine serum albumin; DAB: diaminobenzidine; EDTA: eathylene diamine tetraacetic acid; EGF: epidermal growth factor; ELISA: enzymelinkedimmunosorbent assay; FITC: fluorescein Isothiocyanate; HE: hematoxylin-eosin staining; HOSE: human ovarian surface epithelium; HRP: hoeseradish peroxidase; HRP/ Anti-M13: horseradish peroxidase conjugated to anti-M13 monoclonal antibody; MTT: methyl thazoy terazolium; PBS: phosphate buffered saline; SP: streptavidin-perosidase; TUNEL: termina deoxynucleotidyl transferasemediated dUTP-biotin nick end labeling assay; VEGF: vascular endothelial growth factor.
}

\section{Competing interests}

The authors declared that there were no conflicts of interest in this work.

\section{Authors' contributions}

ZC and KJL carried out the molecular genetic experiments, participated in the sequence alignment, and drafted the manuscript. WXX performed the immunoassays. WW performed sequence alignment. JWY and ZC participated in research design and performed the statistical analysis. KJL conceived the study, research design, study coordination and helped to draft the manuscript. All authors read and approved the final version of this manuscript.

\section{Acknowledgements}

This work was supported in part by grants from Natural Science Foundation of Guangdong Province, China (\#S2012010009341) and Bureau of Science and Technology of Guangzhou Municipality, China (\#11A53150713).

\footnotetext{
Author details

'Department of Obstetrics and Gynecology, Maternity and Children's Healthcare Hospital of Foshan, Foshan 528000Guangdong, China. 2Department of Obstetrics and Gynecology, Guangzhou First People's Hospital, Guangzhou Medical University, Guangzhou 510180, China. ${ }^{3}$ Foshan Hospital of TCM, Foushan 52800, China.
}

Received: 21 July 2015 Accepted: 1 November 2015

Published online: 10 November 2015

\section{References}

1. Gilbert L, Basso O, Sampalis J, Karp I, Martins C, Feng J, et al. Assessment of symptomatic women for early diagnosis of ovarian cancer: results from the prospective DOvE pilot project. Lancet Oncol. 2012;13(3):285-91. doi:10.1016/s1470-2045(11)70333-3.

2. Hu J, Shao S, Song Y, Zhao J, Dong Y, Gong L, et al. Hepatocyte growth factor induces invasion and migration of ovarian cancer cells by decreasing the expression of E-cadherin, beta-catenin, and caveolin-1. Anatomical record (Hoboken, NJ : 2007). 2010;293(7):1134-9. doi:10.1002/ar.21147.

3. Hernandez L, Hsu SC, Davidson B, Birrer MJ, Kohn EC, Annunziata CM. Activation of NF-kappaB signaling by inhibitor of NF-kappaB kinase beta increases aggressiveness of ovarian cancer. Cancer Res. 2010;70(10):4005-14. doi:10.1158/0008-5472.can-09-3912.

4. Kuhn E, Kurman RJ, Shih IM. Ovarian Cancer Is an Imported Disease: Fact or Fiction? Current obstetrics and gynecology reports. 2012;1(1):1-9. doi:10.1007/s13669-011-0004-1.

5. Yap TA, Carden CP, Kaye SB. Beyond chemotherapy: targeted therapies in ovarian cancer. Nat Rev Cancer. 2009;9(3):167-81. doi:10.1038/nrc2583.

6. Sudo T. Molecular-targeted therapies for ovarian cancer: prospects for the future. Int J Clin Oncol. 2012;17(5):424-9. doi:10.1007/s10147-012-0461-1.

7. Matei D, Fang F, Shen C, Schilder J, Arnold A, Zeng Y, et al. Epigenetic resensitization to platinum in ovarian cancer. Cancer Res. 2012;72(9):2197-205. doi:10.1158/0008-5472.can-11-3909.

8. Jelovac D, Armstrong DK. Recent progress in the diagnosis and treatment of ovarian cancer. CA Cancer J Clin. 2011;61(3):183-203. doi:10.3322/caac.20113.

9. Kunos CA, Sill MW, Buekers TE, Walker JL, Schilder JM, Yamada SD, et al. Low-dose abdominal radiation as a docetaxel chemosensitizer for recurrent epithelial ovarian cancer: a phase I study of the Gynecologic Oncology Group. Gynecol Oncol. 2011;120(2):224-8. doi:10.1016/j.ygyno.2010.10.018.

10. Sugiyama T, Konishi I. Emerging drugs for ovarian cancer. Expert Opin Emerg Drugs. 2008;13(3):523-36. doi:10.1517/14728214.13.3.523.

11. Pande J, Szewczyk MM, Grover AK. Phage display: concept, innovations, applications and future. Biotechnol Adv. 2010;28(6):849-58. doi:10.1016/ j.biotechadv.2010.07.004.

12. Han LY, Kipps E, Kaye SB. Current treatment and clinical trials in ovarian cancer. Expert Opin Investig Drugs. 2010;19(4):521-34. doi:10.1517/ 13543781003647966.

13. Zhao Z, Lu P, Zhang H, Xu H, Gao N, Li M, et al. Nestin positively regulates the Wnt/beta-catenin pathway and the proliferation, survival and invasiveness of breast cancer stem cells. Breast cancer research : BCR. 2014;16(4):408. doi:10.1186/s13058-014-0408-8.

14. Tonelli RR, Colli W, Alves MJ. Selection of binding targets in parasites using phage-display and aptamer libraries in vivo and in vitro. Front Immunol. 2012;3:419. doi:10.3389/fimmu.2012.00419.

15. Deutscher SL. Phage display in molecular imaging and diagnosis of cancer. Chem Rev. 2010;110(5):3196-211. doi:10.1021/cr900317f.

16. Phan T, Nguyen HD, Goksel H, Mocklinghoff S, Brunsveld L. Phage display selection of miniprotein binders of the Estrogen Receptor. Chem Commun (Camb). 2010;46(43):8207-9. doi:10.1039/c0cc02727h.

17. Helms BA, Reulen SW, Nijhuis S, de Graaf-Heuvelmans PT, Merkx M, Meijer EW. High-affinity peptide-based collagen targeting using synthetic phage mimics: from phage display to dendrimer display. J Am Chem Soc. 2009;131(33):11683-5. doi:10.1021/ja902285m.

18. Staquicini Fl, Sidman RL, Arap W, Pasqualini R. Phage display technology for stem cell delivery and systemic therapy. Adv Drug Deliv Rev. 2010;62(12):1213-6. doi:10.1016/j.addr.2010.09.014.

19. Matsuo AL, Tanaka AS, Juliano MA, Rodrigues EG, Travassos LR. A novel melanoma-targeting peptide screened by phage display exhibits antitumor activity. Journal of molecular medicine (Berlin, Germany). 2010;88(12):1255-64. doi:10.1007/s00109-010-0671-9.

20. Whitney M, Crisp JL, Olson ES, Aguilera TA, Gross LA, Ellies LG, et al. Parallel in vivo and in vitro selection using phage display identifies proteasedependent tumor-targeting peptides. J Biol Chem. 2010;285(29):22532-41. doi:10.1074/jbc.M110.138297.

21. Hu S, Guo X, Xie H, Du Y, Pan Y, Shi Y, et al. Phage display selection of peptides that inhibit metastasis ability of gastric cancer cells with high 
liver-metastatic potential. Biochem Biophys Res Commun. 2006;341(4):964-72. doi:10.1016/j.bbrc.2006.01.047.

22. Lawrenson K, Grun B, Benjamin E, Jacobs IJ, Dafou D, Gayther SA. Senescent fibroblasts promote neoplastic transformation of partially transformed ovarian epithelial cells in a three-dimensional model of early stage ovarian cancer. Neoplasia. 2010;12(4):317-25.

23. Ehrlich GK, Berthold W, Bailon P. Phage display technology. Affinity selection by biopanning. Methods in molecular biology (Clifton, $\mathrm{NJ}$ ). 2000;147:195-208. doi:10.1385/1-59259-041-1:195.

24. Wang J, Liu Y, Zheng Y, Liao KX, Lin F, Wu CT, et al. Screening peptides binding specifically to colorectal cancer cells from a phage random peptide library. Asian Pacific journal of cancer prevention : APJCP. 2012;13(1):377-81.

25. Beck S, Jin X, Yin J, Kim SH, Lee NK, Oh SY, et al. Identification of a peptide that interacts with Nestin protein expressed in brain cancer stem cells. Biomaterials. 2011:32(33):8518-28. doi:10.1016/j.biomaterials.2011.07.048.

26. Castel G, Chteoui M, Heyd B, Tordo N. Phage display of combinatorial peptide libraries: application to antiviral research. Molecules (Basel, Switzerland). 2011;16(5):3499-518. doi:10.3390/molecules16053499.

27. Askoxylakis V, Zitzmann-Kolbe S, Zoller F, Altmann A, Markert A, Rana S, et al. Challenges in optimizing a prostate carcinoma binding peptide, identified through the phage display technology. Molecules (Basel, Switzerland). 2011;16(2):1559-78. doi:10.3390/molecules16021559.

28. Zhang L, Yin G, Yan D, Wei Y, Ma C, Huang Z, et al. in vitro screening of ovarian tumor specific peptides from a phage display peptide library. Biotechnol Lett. 2011;33(9):1729-35. doi:10.1007/s10529-011-0634-4.

29. Taddei ML, Parri M, Angelucci A, Bianchini F, Marconi C, Giannoni E, et al. EphA2 induces metastatic growth regulating amoeboid motility and clonogenic potential in prostate carcinoma cells. Mol Cancer Res. 2011;9(2):149-60. doi:10.1158/1541-7786.mcr-10-0298.

30. Darnel AD, Behmoaram E, Vollmer RT, Corcos J, Bijian K, Sircar K, et al. Fascin regulates prostate cancer cell invasion and is associated with metastasis and biochemical failure in prostate cancer. Clin Cancer Res. 2009;15(4):1376-83. doi:10.1158/1078-0432.ccr-08-1789.

31. Masoumi Moghaddam S, Amini A, Morris DL, Pourgholami MH. Significance of vascular endothelial growth factor in growth and peritoneal dissemination of ovarian cancer. Cancer Metastasis Rev. 2012;31(1-2):143-62. doi:10.1007/s10555-011-9337-5.

32. Huang C, Huang R, Chang W, Jiang T, Huang K, Cao J, et al. The expression and clinical significance of pSTAT3, VEGF and VEGF-C in pancreatic adenocarcinoma. Neoplasma. 2012;59(1):52-61. doi:10.4149/neo_2012_007.

\section{Submit your next manuscript to BioMed Central and take full advantage of:}

- Convenient online submission

- Thorough peer review

- No space constraints or color figure charges

- Immediate publication on acceptance

- Inclusion in PubMed, CAS, Scopus and Google Scholar

- Research which is freely available for redistribution

Submit your manuscript at www.biomedcentral.com/submit 